\title{
Accurate nighttime blood pressure monitoring with less sleep disturbance
}

\author{
Naoko Tomitani ${ }^{1} \cdot$ Satoshi Hoshide ${ }^{1} \cdot$ Kazuomi Kario $^{1}$ \\ Received: 10 August 2021 / Revised: 15 August 2021 / Accepted: 16 August 2021 / Published online: 21 September 2021 \\ (C) The Japanese Society of Hypertension 2021
}

Nocturnal hypertension has been reported to be a strong risk factor for cardiovascular events [1-4], and the importance of nighttime blood pressure (BP) control for hypertension management has been recognized. Ambulatory BP monitoring (ABPM), which typically measures BP every 15-30 min throughout the day and night, has been considered the gold standard for measuring nighttime BP during sleep. Although considerable evidence has been accumulated from ABPM, its use is not widespread, partly due to the discomfort and disturbance of daily activities and sleep. Recently, home BP monitoring (HBPM) devices equipped with timer functions allowing automatic measurement during sleep have been developed. However, with either method, some individuals feel discomfort and experience sleep disturbance resulting from the frequent brachial cuff inflation of BP measurement during sleep.

Sleep disturbance caused by nighttime BP measurement can lead to the following problems: (1) inaccurate BP assessment due to arousal or sleep deprivation (higher than usual nighttime BP value); and (2) reduced adherence of patients to repeat nighttime BP monitoring (i.e., failure to follow hypertension treatment or to monitor nighttime $\mathrm{BP}$ over multiple nights). In terms of the former problem, a study by Verdecchia et al. assessing perceived sleep quality during overnight ABPM monitoring in 2934 untreated hypertensive subjects found that nighttime BP increased and its prognostic significance decreased in subjects with $\geq 2 \mathrm{~h}$ sleep deprivation induced by cuff inflation [5]. These findings indicate that sleep disturbance and lower than usual sleep quality may lead to underestimation of cardiovascular risk, or perhaps, overdiagnosis. In addition, the Japan Morning Surge-Home Blood Pressure (J-HOP) nocturnal

Naoko Tomitani

tomitani.n@jichi.ac.jp

1 Division of Cardiovascular Medicine, Department of Medicine, Jichi Medical University School of Medicine, Tochigi, Japan
BP study showed the importance of considering both sleep duration and nighttime BP in cardiovascular risk assessment; that is, sleep hygiene is important for implementing cardiovascular disease prevention strategies in patients with hypertension [6]. Related to the latter problem, some previous studies revealed that most subjects who underwent nighttime BP monitoring complained of sleep disturbance [7]. Individuals who experienced reduced sleep quality during nighttime BP measurement may be reluctant to repeat nighttime BP measurements. Therefore, reducing sleep disturbance caused by nighttime BP measurement is indispensable for hypertension management throughout the day and night.

One simple approach to reduce sleep disturbance is to reduce the frequency of BP measurements with cuff inflation per night; however, this will naturally lead to a decrease in the accuracy of nocturnal hypertension diagnoses. Yang et al. investigated the number of BP measurements required to obtain an estimate of mean BP during sleep similar to that obtained by a full night of ABPM in 4277 participants from the International Database on Ambulatory Blood Pressure in Relation to Cardiovascular Outcomes (IDACO) study [8]. They found that 3-4 BP readings during sleep are the minimum requirement to estimate the mean BP from fullnight readings. Moreover, a recent study by Jaeger et al. examined the timing of a limited number of BP measurements to obtain an accurate estimate of mean BP from a full night of ABPM using data from 621 participants of the Jackson Heart study (mean age: $58.8 \pm 10.5$ years, mean asleep SBP: $120 \pm 14.7 \mathrm{mmHg}$ ) and 458 participants of the Coronary Artery Risk Development in Young Adults study (mean age: $54.7 \pm 3.7$ years, mean asleep SBP: $111 \pm 15.1$ $\mathrm{mmHg}$ ), who slept $>5 \mathrm{~h}$ and recorded $>1$ valid asleep BP measurement every $30 \mathrm{~min}$ between midnight and 5:00 AM during their ABPM assessment [9]. They sampled 2-4 BP measurements at specific times from a full night of ABPM readings, and a total of 74 sampling approaches were evaluated for diagnostic accuracy of nocturnal hypertension (defined by a mean nighttime $\mathrm{SBP} \geq 120 \mathrm{mmHg}$ or mean 


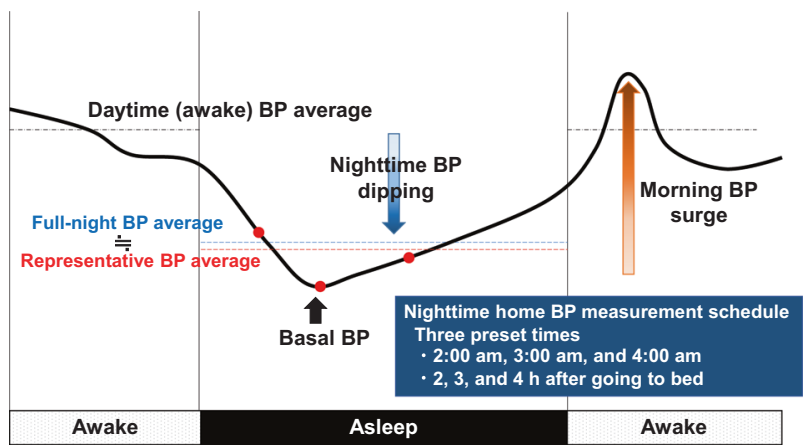

Fig. 1 Average of representative nighttime BP values. The red dots show the sampling BP values. The blue dashed line shows the fullnight BP average value. The red dashed line shows the representative $\mathrm{BP}$ average value (average of the sampling values). BP blood pressure

$\mathrm{DBP} \geq 70 \mathrm{mmHg}$ ) (i.e., Kappa statistics) in comparison to the full-night average and the mean absolute difference. The highest kappa statistic of 0.84 and the lowest mean absolute difference for estimating mean SBP during sleep resulted from sampling $\mathrm{BP}$ at $1,2,4$, and $5 \mathrm{~h}$ after falling asleep. In addition, measuring $\mathrm{BP}$ at 2, 3, and $4 \mathrm{~h}$ after falling asleep, an approach applied for some nocturnal HBPM studies, also showed a high Kappa value of 0.81. BP sampling at 2, 3, and $4 \mathrm{~h}$ after midnight (i.e., 2, 3, and 4 o'clock), which is another approach applied for some nocturnal HBPM studies, showed a lower Kappa value of 0.77 .

In healthy subjects, nighttime BP decreases by 10-20\% from daytime BP, with a decline to the basal BP (lowest BP during sleep) and a rise to regain daytime BP levels before awakening [2]. If sampling points could coincide with the decline, basal, and rise phases, their average (representative $\mathrm{BP}$ average) would be comparable to the full-night average (Fig. 1). In Jaeger's data, SBP measured 3-4 h after falling asleep was basal. Those sampling points (i.e., 1, 2, 4, and 5 $\mathrm{h}$, or 2,3 , and $4 \mathrm{~h}$ after falling asleep) tended to coincide with the period when BP was in decline, basal, and rising, and their average would be comparable to the full-night average. Our recent study, in which nighttime BP was measured hourly using a wrist-type nocturnal HBPM device in 50 hypertensive patients (mean age: $68.9 \pm 11.3$ years, mean asleep SBP: $116.4 \pm 12.3 \mathrm{mmHg}$ ), showed a similar $\mathrm{BP}$ trend, with the basal BP at the 3rd hourly measurement after bedtime (i.e., 2-3 h after falling asleep) [10]. In our study, the mean of three fixed time measurements at 2, 3, and 4 o'clock was comparable to that of hourly full-night measurements, but the mean of the $2 \mathrm{nd}$, 3rd, and 4th hourly measurements after falling asleep was not. As Jaeger et al. noted as a limitation of their study, their results need to be interpreted with caution and may not apply to all individuals, including short sleepers and older adults.

In a comparison study of nighttime BP measured by brachial HBPM and ABPM using the data of 1005 participants from the J-HOP study, HBPM-defined nocturnal hypertension (nighttime home $\mathrm{SBP} \geq 120 \mathrm{mmHg}$ ) was associated with an increased risk of future cardiovascular events, but there was no significant association with ABPM-defined nocturnal hypertension (nighttime ambulatory $\mathrm{SBP} \geq 120 \mathrm{mmHg}$ ) [11]. In this study, nighttime home BP was measured at 2, 3, and 4 o'clock over an average of 8.9 nights $(25.1 \pm 12.8$ nighttime measurements), and ABPM nighttime BP was measured every $30 \mathrm{~min}$ over one night $(14.2 \pm 2.9$ nighttime measurements). ABPM typically records nighttime BP for only one $24 \mathrm{~h}$ period, which may result in the misestimation of true nighttime measurements. Additionally, HBPM records BP repeatedly over several days with an increased number of measurements and thus may provide more accurate nighttime BP measurements and prognostic power.

On the other hand, the advantage of ABPM is its ability to measure BP throughout the day and night and to assess diurnal BP variation, such as nighttime BP dipping and morning BP surge, which are known to be strong risk factors for cardiovascular events (Fig. 1) [12-14]. Jaeger et al. indicated that fewer BP measurements taken at specific timings could increase the likelihood of patients agreeing to have their nighttime BP assessed over multiple nights. Their results could be interpreted to suggest that ABPM with fewer nighttime measurements might be useful for monitoring $24 \mathrm{~h} \mathrm{BP}$ over multiple days to obtain accurate nighttime BP with less sleep disturbance. However, there is no evidence regarding whether nighttime BP dipping and morning BP surge calculated from fewer nighttime readings could predict future cardiovascular risk. Further investigation of the accuracy of diurnal BP variability indices and the patient acceptability of multiple night $\mathrm{BP}$ measurements using ABPM with fewer nighttime measurements is warranted.

Another approach to reduce sleep disturbance is the development of a new device with less restriction, discomfort, and measurement noise. A newly developed wristtype nocturnal HBPM device (HEM-9601T; Omron Healthcare, Kyoto, Japan) reduces the discomfort caused by cuff inflation and measurement noise. Our study group has confirmed the BP values measured by the HEM-9601T to be reliable under both laboratory conditions [15] and sleeping conditions at home [16]. In our study, which measured BP hourly with the HEM-9601T during sleep (average 7.2 measurements per night), only a few of 50 study participants reported nocturnal awakening caused by the HEM-9601T measurement [16]. Additionally, in a previous study by Imai et al. comparing the acceptability of wrist-cuff and upper arm-cuff nocturnal HBPM, sleep disturbance was reported to be $<20 \%$ with the wrist device, substantially less than with the upper arm device (70\%) [17].

A growing body of evidence on nighttime $\mathrm{BP}$ has been supported by the development of technologies such as smaller 
and lighter ABPM devices [18], and HBPM devices with nocturnal BP measurement function; however, sleep disturbance caused by nocturnal BP measurements remains a bottleneck to widespread assessment of nighttime BP. The findings of Jaeger et al. and the development of new device technology should support the acquisition of higher accuracy nighttime BP with less sleep disturbance.

\section{Compliance with ethical standards}

Conflict of interest $\mathrm{KK}$ has received research grants from Omron Healthcare and A\&D Co. The other authors have no competing interests.

Publisher's note Springer Nature remains neutral with regard to jurisdictional claims in published maps and institutional affiliations.

\section{References}

1. Boggia J, Li Y, Thijs L, Hansen TW, Kikuya M, BjorklundBodegard K, et al. International Database on Ambulatory blood pressure monitoring in relation to Cardiovascular Outcomes i. Prognostic accuracy of day versus night ambulatory blood pressure: a cohort study. Lancet. 2007;370:1219-29.

2. Kario K. Nocturnal hypertension: new technology and evidence. Hypertension. 2018;71:997-1009.

3. Kario K, Kanegae H, Tomitani N, Okawara Y, Fujiwara T, Yano $\mathrm{Y}$, et al. Nighttime blood pressure measured by home blood pressure monitoring as an independent predictor of cardiovascular events in general practice. Hypertension. 2019; 73:1240-8.

4. Hoshide S, Kanegae H, Kario K. Nighttime home blood pressure as a mediator of $\mathrm{N}$-terminal pro-brain natriuretic peptide in cardiovascular events. Hypertens Res. 2021;44:1138-46.

5. Verdecchia P, Angeli F, Borgioni C, Gattobigio R, Reboldi G. Ambulatory blood pressure and cardiovascular outcome in relation to perceived sleep deprivation. Hypertension. 2007; 49:777-83.

6. Kario K, Hoshide S, Nagai M, Okawara Y, Kanegae H. Sleep and cardiovascular outcomes in relation to nocturnal hypertension: the J-HOP Nocturnal Blood Pressure Study. Hypertens Res. 2021. June 30.

7. Asayama K, Fujiwara T, Hoshide S, Ohkubo T, Kario K, Stergiou GS, et al. International Expert Group of Nocturnal Home Blood P. Nocturnal blood pressure measured by home devices: evidence and perspective for clinical application. J Hypertens. 2019;37:905-16.

8. Yang WY, Thijs L, Zhang ZY, Asayama K, Boggia J, Hansen $\mathrm{TW}$, et al. behalf of the International Database; on Ambulatory blood pressure in relation to Cardiovascular Outcomes (IDACO) Investigators. Evidence-based proposal for the number of ambulatory readings required for assessing blood pressure level in research settings: an analysis of the IDACO database. Blood Press. 2018;27:341-50.

9. Jaeger BC, Akinyelure OP, Sakhuja S, Bundy JD, Lewis CE, Yano Y, et al. Number and timing of ambulatory blood pressure monitoring measurements. Hypertens Res. 2021;Aug 11.

10. Tomitani N, Kanegae H, Kario K. Comparison of nighttime measurement schedules using a wrist-type nocturnal home blood pressure monitoring device. J Clin Hypertens. 2021;23:1144-9.

11. Mokwatsi GG, Hoshide S, Kanegae H, Fujiwara T, Negishi K, Schutte AE, et al. Direct comparison of home versus ambulatory defined nocturnal hypertension for predicting cardiovascular events: the Japan Morning Surge-Home Blood Pressure (J-HOP) Study. Hypertension. 2020;76:554-61.

12. Kario K, Pickering TG, Umeda Y, Hoshide S, Hoshide Y, Morinari $\mathrm{M}$, et al. Morning surge in blood pressure as a predictor of silent and clinical cerebrovascular disease in elderly hypertensives: a prospective study. Circulation. 2003;107:1401-6.

13. Salles GF, Reboldi G, Fagard RH, Cardoso CR, Pierdomenico $\mathrm{SD}$, Verdecchia $\mathrm{P}$, et al. Investigators A-H. prognostic effect of the nocturnal blood pressure fall in hypertensive patients: the ambulatory blood pressure collaboration in patients with hypertension (ABC-H) meta-analysis. Hypertension. 2016;67:693-700.

14. Kario K, Hoshide S, Mizuno H, Kabutoya T, Nishizawa M, Yoshida T, et al. Nighttime blood pressure phenotype and cardiovascular prognosis: practitioner-based nationwide JAMP study. Circulation. 2020;142:1810-20.

15. Kuwabara M, Harada K, Hishiki Y, Ohkubo T, Kario K, Imai Y. Validation of a wrist-type home nocturnal blood pressure monitor in the sitting and supine position according to the ANSI/AAMI/ ISO81060-2:2013 guidelines: Omron HEM-9601T. J Clin Hypertens. 2020;22:970-8.

16. Kario K, Tomitani N, Iwashita C, Shiga T, Kanegae H. Simultaneous self-monitoring comparison of a supine algorithm-equipped wrist nocturnal home blood pressure monitoring device with an upper arm device. J Clin Hypertens. 2021;23:793-801.

17. Imai Y, Asayama K, Fujiwara S, Saito K, Sato H, Haga T, et al. Development and evaluation of a home nocturnal blood pressure monitoring system using a wrist-cuff device. Blood Press Monit. 2018;23:318-26.

18. Kario K, Hoshide S, Saito K, Sato K, Hamasaki H, Suwa H, et al. Validation of the TM-2441 ambulatory blood pressure measurement device according to the ISO 81060-2: 2013 standard. Blood Press Monit. 2019;24:38-41. 